\title{
Le « roman-essai » (1934-1947). Un précurseur de l'essai littéraire au Québec?
}

\author{
David Décarie \\ Université de Moncton \\ Marie-Frédérique Desbiens \\ Fonds de recherche du Québec - Société et culture
}

\begin{abstract}
Résumé
Cet article vise à décrire un nouveau type de roman qui apparaît dans les années 1930 et 1940 au Québec et que nous proposons de nommer « romans-essais ». Notre article repère dans 9 œuvres hybrides tenant à la fois du roman et de l'essai de nombreuses caractéristiques communes (tantôt thématiques, tantôt formelles). Cet article rapproche de plus le roman-essai de la forme plus spécifique de l'essai (parfois appelée « essai littéraire ») qui a connu un développement important au Québec dans les années 1950 et 1960 et émet l'hypothèse qu'il s'agirait d'un précurseur de ce genre.
\end{abstract}

\begin{abstract}
This article aims to describe a new type of novel that appeared in the 1930's and 1940's in Quebec which we propose calling essay-novels. Our article identifies nine hybrid works that draw numerous characteristics (some thematic, some in form) from both novels and essays. This article relates the essay-novel to the more specific form of essay (sometimes called "literary essay") that blossomed in the 1950's and 1960's in Quebec and proposes that it is a precursor to this genre.
\end{abstract}


DAVID DÉCARIE et MARIE-FRÉDÉRIQUE DESBIENS, « Le "roman-essai” (19341947). Un précurseur de l'essai littéraire au Québec?»

Dans le cadre des recherches que nous menons avec le groupe de recherche La vie littéraire au Québec, nous avons étudié les 111 romans $^{1}$ publiés des années 1934 à 1947 en cherchant à jeter un regard neuf sur ces œuvres. Des surprises de taille se sont révélées au fil de nos lectures : le roman urbain s'avère beaucoup plus présent que prévu (Décarie, 2016); à côté des romans de la terre, moins nombreux qu'on aurait pu le croire, se profile un corpus important de romans de la forêt. Une des surprises les plus marquantes est toutefois la lecture de nombreuses œuvres singulières, novatrices au niveau formel et qui échappent aux différents classements de l'histoire littéraire. En examinant et réexaminant ces œuvres curieuses, nous avons remarqué qu'elles partagent de nombreuses caractéristiques, à commencer par une forme hybride qui tient à la fois du roman et de l'essai. Nous tenterons, dans cet article, de décrire ce nouveau type de roman, que nous proposons de nommer « romans-essais $»^{2}$.

\footnotetext{
${ }^{1}$ Cette liste est établie à partir du catalogue de Bibliothèque et Archives nationales du Québec et des volume II et III du Dictionnaire des œeuvres littéraires du Québec: Maurice Lemire (dir.), Dictionnaire des oeuvres littéraires du Québec. 1900 à 1939, 2e édition revue, corrigée et mise à jour, Montréal, Fides, 1987, vol. II; Maurice Lemire (dir.), Dictionnaire des ouvres littéraires du Québec. 1940 à 1959, 2e édition revue, corrigée et mise à jour, Montréal, Fides, 1995, vol. III. Celle-ci ne comprend toutefois pas les romans d'auteurs étrangers ou en traduction, les romans en fascicules, les romans sentimentaux, les romans pour la jeunesse, les rééditions des périodes précédentes ni les rééditions d'un même titre durant la période.

${ }^{2}$ Nous entendrons, dans un premier temps, le terme « essai » dans sa forme la plus générale de « texte réflexif ».
} 
Dans une présentation des méthodes de l'équipe de recherche de La vie littéraire au Québec, Lucie Robert souligne :

l'importance de susciter des recherches nouvelles sur les terrains moins pourvus. En effet, s'il est vrai que l'histoire littéraire est « constituée par emprunt et capitalisation de matériaux explicatifs antérieurement constitués », il est aussi vrai qu'un travail de synthèse comme le nôtre doive combler « les lacunes et les déséquilibres de la recherche laissée à elle-même $»^{3}$. (2011, p. 102-103)

Cette méthode pose toutefois quelques difficultés, surtout dans le cadre d'un article. L'ambition de son objet (proposer des «recherches nouvelles»: ici décrire un genre nouveau) se heurte aux difficultés propres aux « terrains moins pourvus ». Il nous faut ainsi embrasser un corpus important (9 romans) composé d'œuvres hétéroclites, majoritairement oubliées, et se démarquant par leur originalité. Il nous faut donc les présenter et les décrire, ce qui limite leur analyse. Pour ajouter à ces difficultés, nous analysons une forme hybride. Notre article ne prétend donc nullement épuiser le sujet qu'il présente4 et doit être envisagé comme une proposition.

Nous nous proposons de montrer que, malgré leur diversité et leur originalité, les romans-essais des années trente et quarante gagnent à être lus conjointement et que ce regroupement jette un éclairage nouveau sur les œuvres, notamment sur les œuvres plus connues. Comme l'analyse des romans de la forêt permet de mieux situer Menaud, maître draveur $^{5}$, celle des romans-essais met en relief de nouvelles caractéristiques des Demi-

\footnotetext{
${ }^{3}$ Les deux citations sont respectivement issues de : Rémy Ponton, «Durkheim et Lanson», Michel Espagne et Michel Werner (dir.), Philologiques, t. I: Contribution à l'histoire des disciplines littéraires en France et en Allemagne au xixe siècle, Paris, Éditions de la Maison des sciences de l'homme, 1990, p. 262 ; et Jean-Claude Payen et Henri Weber (dir.), «Introduction», Manuel d'histoire littéraire de la France, t. I: Des origines à 1600, Paris, Éditions sociales, 1971, p. 12.

${ }^{4}$ Faute de place, nous ne pourrons pas retracer l'évolution de ce genre ou situer celui-ci au sein de la constellation des autres types de romans des années trente et quarante, à commencer par le roman urbain et le roman psychologique avec lesquels les romans-essais ont de nombreuses affinités.

${ }^{5}$ Voir le tome VII de La vie littéraire au Québec, à paraître.
} 
DAVID DÉCARIE et MARIE-FRÉDÉRIQUE DESBIENS, « Le “roman-essai” (19341947). Un précurseur de l'essai littéraire au Québec?»

civilisés. Notre article repose, pour l'essentiel, sur le repérage de huit caractéristiques communes (tantôt thématiques, tantôt formelles) de ces œuvres. Nous avons étayé ce repérage par une étude de la réception de ces œuvres.

Nous présenterons, en premier lieu, ce corpus d'œuvres hétéroclites en montrant que la plupart de celles-ci - première caractéristique commune - manipulent des idées modernes qui remettent en question le statu quo ayant cours dans la société canadienne-française de l'époque. D'abord l'œuvre de tireurs solitaires, comme Jean-Charles Harvey, Georges Bugnet, Jeannine Lavallée et Thérèse Tardif, le roman-essai donne ensuite lieu, après 1944 et autour de la revue Amérique française, aux expérimentations littéraires de Pierre Baillargeon, François Hertel et Jean Simard. Nous examinerons, en second lieu, le renouvellement de la forme romanesque que ces œuvres mettent en place en explorant quatre caractéristiques communes : soit l'hybridité, l'autoréflexivité, le caractère autobiographique et la dissolution de l'intrigue. Nous explorerons ensuite les particularités de leur dimension essayistique en analysant trois dernières caractéristiques communes : soit leur fragmentation (formelle et thématique); leur caractère négatif et la mise à distance des réflexions. Le repérage de ces diverses caractéristiques nous permettra, en conclusion, de rapprocher le roman-essai de la forme plus spécifique de l'essai, parfois appelée « essai littéraire » et de se demander s'il ne s'agirait pas en fait d'un précurseur de ce genre au Québec. 
Www.revue-analyses.org, vol. 15, nº1, printemps-été 2020, p. 26-53.

\section{Neuf romans-essais}

Dans les années 1920-30, le roman idéologique ou roman à thèse est largement pratiqué dans ses variantes nationalistes (L'Appel de la race publié en 1922 par Lionel Groulx sous le pseudonyme d'Alonié de Lestres) ou du terroir (L'appel de la terre de Damase Potvin publié en 1919). Si le modèle demeure bien présent dans les années 1930 et 1940, il tend néanmoins à se diversifier. L'idéologie de la terre et le nationalisme continuent d'inspirer les romancières et romanciers (Marilyne, pseudonyme d'Aline Séguin-Le Guiller, fait paraître Le flambeau sacré en 1944; Allie de Joseph Lallier, est publié en 1936), mais les thèses défendues s'élargissent afin de mieux correspondre aux réalités du temps : lutte contre le divorce (Madame Després de Jean Véron, pseudonyme de l'abbé Eddie Hamelin, 1934); prévention de la tuberculose (Sanatorium de Paul Dufault, 1938); dénonciation du travail des femmes (Trahison de Geneviève de Francheville, 1946) ou de l'immigration (Zirska, immigrante inconnue de Jean-Marie Carrette 1947). La guerre donne aussi lieu à deux thèses opposées : traitant de la Première Guerre mondiale, Adolphe Brassard écrit un roman pacifiste, Mémoires d'un soldat inconnu (1939), tandis que, dans La conscience de Pierre Laubier (1942), Oscar Massé montre au contraire les dangers d'un refus de la conscription. Défendant pour la plupart des valeurs réactionnaires, ces romans à thèse n'innovent guère au plan de la forme.

Dans les années 1930 et 1940 paraissent toutefois des œuvres singulières qui ont en commun de proposer des idées contestataires dans une forme inédite qui mélange le roman et l'essai. La veine du roman-essai connaît des débuts fracassants avec la publication, en avril 1934 aux Éditions du Totem, du roman Les Demi-civilisés de Jean-Charles Harvey, qui détourne le roman québécois des thèses qui lui étaient traditionnellement associées. Les idées 
DAVID DÉCARIE et MARIE-FRÉDÉRIQUE DESBIENS, « Le “roman-essai” (19341947). Un précurseur de l'essai littéraire au Québec?»

sont au cœur de ce roman noir et pessimiste qui raconte la création, par le protagoniste Max Hubert, d'une revue de combat, le Vingtième siècle, une « entreprise de libération » (Harvey, 1988 [1934], p. 149) qui récolte, après trois ans d'efforts, l'appui de « douze à quinze milles civilisé » (1988 [1934], p. 149):

Pour la première fois, en ce pays de l'impersonnel et de l'artifice, où seule la pensée officielle avait eu droit de cité, paraissait une publication vraiment libre, ouverte à toutes les opinions sensées, débarrassée des préjugés dont vivaient depuis un siècle et demi, les neuf dixièmes des gens. » (1988 [1934], p. 149)

Véritable pamphlet, l'œuvre s'attaque aux élites bourgeoises et à la mainmise de la religion sur le peuple, prône la modernisation de la société et défend la liberté sous toutes ses formes, tout particulièrement dans le domaine littéraire. Le Vingtième siècle se porte, par exemple, à la défense de l'auteur fictif Hermann Lillois, attaqué pour cause d'immoralité.

Un second roman-essai, Siraf, de Georges Bugnet, paraît la même année aux Éditions du Totem. Bugnet signe une œuvre singulière qui, davantage encore que le roman d'Harvey, fait la part belle à l'essai. Le narrateur homodiégétique (et se présentant comme l'auteur même) est un adepte des lumières qui loue les progrès de la civilisation, allant jusqu'à chanter les réalisations de la révolution russe. Celui-ci fait toutefois la rencontre de deux esprits extraterrestres, Siraf et Karis, qui n’auront de cesse de déboulonner ses idées.

Koshawika, l'œuvre de Jeannine Lavallée, qui paraît aux éditions Rénovation deux ans plus tard, amalgame également les formes du roman et de l'essai, mais de manière plus franche, plus nette. En effet, Koshawika se divise distinctement en deux parties : un court « roman », proche du conte, est suivi d'un essai qui en constitue une glose assez libre. La partie romanesque se déroule dans une maison de plaisance des Laurentides nommée 
Www.revue-analyses.org, vol. 15, nº1, printemps-été 2020, p. 26-53.

Koshawika et raconte la mystérieuse disparition de la jeune Olivette Dansereau. Celle-ci sombre dans la folie à la suite du décès de sa mère (qu'elle aurait provoqué en la maudissant) et du second mariage de son père avec une de ses camarades de classe. L'essai de la seconde partie vise à expliquer le sens de ce drame en montrant comment le Québec fait le malheur de sa jeunesse : « Madame Dansereau, c’est notre âme française. Naomie Scott n’est pas sans analogie avec notre âme anglicisée, Olivette, c'est notre évolution. Elle est la parfaite image de nos talents qui ne peuvent s'épanouir » (Lavallée, 1936, p. 99). Comme chez Harvey, la modernité de Lavallée passe, dans le versant essayistique, par une peinture au noir du Québec et de ses élites bourgeoises, dont elle dénonce le snobisme, l'esprit de chapelle et le peu de goût pour l'instruction et pour les arts et lettres. Elle déplore également le retard des Canadiens français dans les domaines des sciences et de l'industrie. Fervente nationaliste, elle demande un drapeau pour le Québec et l'abandon du mouton comme emblème national : « Si l'agneau est le symbole de la docilité, il a d'autres attributs plus ou moins méprisables, Panurge l'a prouvé » (1936, p. 154). La dernière partie est même un appel au «grand réveil » : « Je crois au grand réveil. Je crois au succès des nôtres par la rénovation de notre esprit critique, de notre mentalité. Je crois en nous, en l'avenir dans le travail » (1936, p. 192).

Thérèse Tardif fait paraître en 1943, aux Éditions de l'Arbre, Désespoir de vieille fille (1943). Comme l'indique l'autrice en exergue, l'œuvre se présente sous forme de « feuillets détachés » que relient le cheminement intérieur de la narratrice confrontée aux dures réalités d'être femme. Celle-ci dénonce le pouvoir de l'homme et de l'église, la soumission qu'elle doit à l'un et à l'autre. Le livre fait réagir Simone Routier qui, sous le pseudonyme de Marie 
DAVID DÉCARIE et MARIE-FRÉDÉRIQUE DESBIENS, « Le “roman-essai” (19341947). Un précurseur de l'essai littéraire au Québec?»

de Villiers, publie la même année une Réponse à Désespoir de vieille fille, qui se veut un pastiche méprisant de l'œuvre visant à amoindrir la portée de ses réflexions contestatrices.

Si ces premiers romans-essais ne semblent pas avoir de suite, quelques œuvres marquantes des années 1940 poursuivent et poussent encore plus loin l'exploration du métissage de l'essai et du roman, celles de François Hertel et Pierre Baillargeon. En 1944, l'auteur du roman d'apprentissage Le Beau risque (1939), François Hertel, fait paraître Anatole Laplante, curieux homme, aux éditions de l'Arbre, œuvre identifiée sur la couverture comme un roman ${ }^{6}$. Pour sa part, Pierre Baillargeon publie l'année suivante les Médisances de Claude Perrin, aux éditions Parizeau, sans mention de genre. En 1947, Hertel et Baillargeon mettent tous deux le point final à leur cycle alors que paraissent le Journal d'Anatole Laplante aux éditions Serge Brousseau et Commerce aux éditions Variétés, Dussault et Péladeau. Cet éditeur publie, la même année, un autre « essai sous forme de récit (Mailhot, 1982, p. 370) »: Félix, livre d'enfant pour adultes de Jean Simard qui sera suivi d'Hôtel de la reine, aux Éditions Variétés, en 1949, année qui voit le départ d'Hertel et Baillargeon en France. Ces trois auteurs ont en commun d'inscrire leurs romans-essais dans des cycles littéraires et de mettre en scène des anti-héros (ou, selon Mailhot, des « misanthropes » [1982, p. 370]) dans des récits ironiques et éclatés qui critiquent la société canadienne-française de l'époque.

\footnotetext{
${ }^{6}$ Celui-ci est la suite du recueil de nouvelles Mondes chimériques paru en 1940 aux éditions Bernard Valiquette. Par le retour de ses personnages principaux, le recueil s'apparente toutefois déjà au roman. C'est dans cette œuvre inclassable qu'apparaît son héros Anatole Laplante, mais également le double et mentor de ce dernier, Charles Lepic.
} 
www.revue-analyses.org, vol. 15, nº1, printemps-été 2020, p. 26-53.

Soulignons que le roman-essai des années 1940, contrairement à celui des années 1930, est écrit par deux auteurs solidement ancré dans l'institution littéraire et en étroite relation avec une nouvelle génération d'écrivaines et d'écrivains. En 1941, Pierre Baillargeon et Roger Rolland, tous deux sortis du Collège Jean-de-Brébeuf fondent la revue Amérique française $^{7}$ où publiera leur ancien professeur, François Hertel. Ce dernier dirigera la revue en 1946 et 1947. Les anti-héros Plante et Perrin apparaissent d'ailleurs dans la revue avant leur parution en volume. Même si la plupart des romans-essais sont aujourd'hui oubliées, il faut reconnaître les tentatives de leurs autrices et auteurs pour renouveler la forme romanesque.

\section{Le renouvellement du roman par l'essai : hybridité, autoréflexivité, caractère} autobiographique et dissolution de l'intrigue ${ }^{8}$

Les romans-essais de notre corpus ont en commun d'avoir voulu sortir des sentiers battus du genre romanesque. Malgré leur diversité et leur caractère hétéroclites, ils possèdent d'importantes ressemblances tant sur le plan formel que thématique. Outre leur caractère contestataire, ces romans possèdent tous, à des degrés divers, une forme hybride.

Signant la seule critique des Demi-civilisés parue avant sa condamnation par le cardinal Villeneuve, Berthelot Brunet est frappé par cette caractéristique : «D’abord, ça n’est

\footnotetext{
${ }^{7}$ Les œuvres de Baillargeon et Hertel participent de l'esthétique forgée dans Amérique Française analysée par Michel Lacroix et Élyse Guay : « Ethos ironique et condescendant, réflexivité du discours, de la poésie et de la fiction, exploration d'une littérature inaccessible au commun et privilégiant le second degré, ces éléments caractéristiques d'Amérique française participent à l'élaboration d'une poétique de la saillie et du paradoxe. Avec elle, une nouvelle figure d'écrivain fait son apparition sur la scène littéraire québécoise, figure composite qui combine les traits du bouffon et du moraliste, l'esprit de recherche et l'esprit critique ». (Guay et Lacroix, 2016, p. 68).

${ }^{8}$ Le terme de « renouvellement » est miné : nous l'entendons ici dans le contexte du Québec et des années 1930 et 1940. De plus, nous ne prétendons pas que les diverses caractéristiques que nous mettons ici de l'avant ne se retrouvent pas également, avant et durant la période, dans d'autres romans. Le « renouvellement » provient selon nous de la combinaison de toutes ces caractéristiques.
} 
DAVID DÉCARIE et MARIE-FRÉDÉRIQUE DESBIENS, « Le “roman-essai” (19341947). Un précurseur de l'essai littéraire au Québec?»

pas un roman, mais un recueil de thèses, de conversations et de rêves » (1934a, p. 4). L'hybridité de l'œuvre lui fait même douter de son appartenance au genre romanesque. Ce flottement générique apparaît au détour de nombreuses critiques : Biron, Dumont et NardoutLafarge définissent ainsi le roman comme une «sorte de pamphlet romanesque en même temps qu'un texte à teneur autobiographique » (2007, p. 252). Formellement, cette hybridité provient notamment de la place que donne Harvey à la revue de combat dans le roman. La nouveauté du procédé n'est guère soulignée puisque l'hybridité du roman est confondue avec le caractère idéologique des romans à thèse de la période précédente évoqué plus haut.

Cette même hybridité apparaît dès la page couverture de Siraf, par la surenchère des sous-titres : «Étranges Révélations »; «Ce qu’on pense de nous par-delà la lune ». L'« étrange récit » décontenance la critique : « livre bizarre », « monstre » (1934b, p. 4), écrit Brunet. La nouveauté de cette forme hybride est toutefois plus facilement assimilable que celle des romans-essais ultérieurs. Les critiques peuvent rattacher celui-ci à des œuvres connues. Brunet remarque, par exemple, qu'avec son œuvre, Bugnet puise à la grande tradition du roman philosophique (l'auteur évoque d'ailleurs Candide). Il exploite également la veine fantaisiste qui a fait la fortune d'un Swift, tandis que le sous-titre renvoie dans le même temps à Cyrano dans sa référence à « L'autre monde », le monde lunaire. Comme il le fait pour le roman d'Harvey, Brunet souligne le caractère plurigénérique de l'œuvre : « Et les neuf ou dix chapitres ne sont qu'essais, voire chroniques et articles de journaux dialogués. Dialogues qui, souvent, ne sont que les monologues de Siraf » (1934b, p. 4). Il reprend même le terme de recueil (« recueil d'essais philosophiques, sociologiques » [1934b, p. 4]). 
Www.revue-analyses.org, vol. 15, nº1, printemps-été 2020, p. 26-53.

Dans la même perspective, le caractère hybride de Koshawika n'a pas d'équivalent dans la littérature québécoise. Si ce roman-essai ne connaît pas une grande fortune, une courte recension de R. D. (sans doute Roger Duhamel), dans L'Action nationale, montre que l'ambivalence entre les deux genres est remarquée sans être encouragée : « Nous croyons que l'auteur aurait tout avantage à négliger la littérature de combat (où elle devient facilement ridicule) pour s'adonner uniquement à la fiction. Sa grande sensibilité est une force pour le romancier; elle est peut-être un écueil pour l'essayiste et le polémiste » (1938, p. 264) ${ }^{9}$.

L’hybridité est également un des moteurs de la création dans Désespoir de vieille fille. Le Jour résume l'entreprise : « Ce n'est pas un roman, ce n’est pas non plus le journal d'une femme, mais le livre tient à la fois de ces deux genres » ([Anonyme], 1934, p. 3). L'œuvre est également très proche de la poésie en prose et le modèle de Rimbaud est très souvent évoqué par Tardif.

Dans les romans de Hertel et Baillargeon, l'hybridité est pareillement omniprésente. Ces « mondes chimériques » constituent de véritables fourre-tout où sont recyclés les écrits les plus divers. L'hybridité de ses œuvres est perceptible dans les efforts que doit faire Hertel pour tenter d'imposer au public le caractère romanesque de sa trilogie « qui n'est nullement un essai, ni des contes, mais un roman fleuve ou simplement rivière, si l'on préfère » (Hertel, 1944, p. 156). Simard n'est pas en reste puisque son Félix est sous-titré : livre d'enfant pour adultes et comporte des illustrations de l'auteur. L'intégration de la forme de l'essai (au sens large) est ainsi commune aux œuvres de notre corpus. L'hybridation de ces œuvres

\footnotetext{
${ }^{9}$ De nombreux critiques, dont Lucien Parizeau (voir 1934, p. 1), recommandent à Harvey de faire le contraire.
} 
DAVID DÉCARIE et MARIE-FRÉDÉRIQUE DESBIENS, « Le “roman-essai” (19341947). Un précurseur de l'essai littéraire au Québec?»

polymorphes et plurigénériques dépasse toutefois largement ces deux genres car elles intègrent de très nombreux autres genres (journal, poésie, dialogues, livre pour enfant, etc.).

Les romans de notre corpus se ressemblent en outre par leur caractère autoréflexif. Ils mettent en effet tous en scène des écrivains. Peut-être parce qu'ils sortent des sentiers battus et qu'ils explorent des formes inédites, tous les autrices et les auteurs sentent le besoin de réfléchir, à l'intérieur même de leurs textes, à leur création pour l'expliquer, la défendre.

Le caractère autoréflexif des Demi-civilisés n'apparaît pleinement que lorsqu'on le considère comme un roman-essai car celui-ci ne raconte pas l'écriture d'un roman mais la mise sur pied d'une revue engagée. Harvey, par ce truchement, réfléchit aux enjeux de son œuvre, et notamment à ses risques. Avec une fascination morbide, il met ainsi en scène l'exclusion sociale dont sera victime son héros pour avoir ouvertement défendu des thèses libertaires. Dans une lettre à Louvigny de Montigny écrite en mai 1934 à Cambridge, le critique Louis Dantin remarque le caractère prophétique du roman d'Harvey:

La catastrophe qui atteint Jean-Charles Harvey est lamentable; mais il m'a tout l'air d'y avoir marché les yeux grands ouverts. Il ne pouvait supposer qu'une attaque contre le clergé si ouverte, si virulente, pût rester sans riposte. Bien plus il avait décrit en détail dans son livre même toute l'aventure qui lui arrive! ... Peut-être n'était-il pas très sage d'aller au-devant de ces coups. (1965, p. 23-24)

Il existe aussi, dans Koshawika, une part d'autoréflexivité évidente. La préface de l'œuvre, intitulée « une opinion autorisée », est assez révélatrice du rapport complexe des deux genres. Il s'agit de la transcription d'une lettre de Crémazie à l'abbé Casgrain prenant position contre le roman au profit des idées. Si l'œuvre d'Harvey met en scène sa mise à l'index, celle de Lavallée prévoit l'indifférence et l'oubli qui lui sont promis. Illustration de la déstructuration de l'édition et du dénuement de écrivains des années trente, son essai 
Www.revue-analyses.org, vol. 15, nº1, printemps-été 2020, p. 26-53.

raconte ainsi l'humiliante expérience d'avoir tenté, sans succès, de vendre de porte en porte son premier essai intitulé Mea Culpa.

L’autoréflexivité est également centrale dans Désespoir de vieille fille : «Vengeance de la nature? Sitôt que je me remets à écrire, mes vêtements se torchonnent. » (Tardif, 1943, p. 28) La thématique de l'amour croise celle de l'écriture lorsque la narratrice évoque, sous le mode de l'ironie, ses relations avec R, un écrivain volage : « R... poursuit la publication par tranche de son premier roman. Une autre femme, un autre chapitre. [...] Pour R..., une femme : une étude, une expérience, un roman » (1943, p. 23; 28). L'amour inspire l'écrivaine : «N'ai-je pas réussi, n'ayant plus rien à vous dire, à remplir huit feuillets de pensées que vos silences avaient suscitées en moi? » (1943, p. 29)

L'écriture est sans cesse au premier plan dans les romans de Baillargeon et Hertel dont le versant essayistique ne porte pas que sur la société, mais également sur la littérature. L'autoréflexivité mène de plus, chez eux, à des procédés pirandelliens où la fiction s'avoue fictionnelle. Hertel termine Anatole Laplante, curieux homme, par exemple, par un chapitre métatextuel intitulé « La danse des personnages » où le narrateur s'adresse à ses lecteurs :

À ce moment de notre récit, puisque tout va mourir et qu'il faudra bientôt mettre le point final, moi au plaisir d'écrire, et vous, lecteur, je n'en doute pas, à la joie de me lire, alors qu'une autre aventure, d'évasion pour vous, de création pour moi, s'achève et que je vous ai enrichi en me vidant, ne convient-il pas de faire le point et de réunir les éléments épars d'une personnalité qui allait s'affirmant, de prendre conscience une fois pour toutes des divers personnages et des événements qui ont de l'extérieur contribué à modeler Laplante et à lui imposer un intérieur qu'il ne voulait pas nécessairement tel ? (1944, p. 155)

L'autoréflexivité entraîne de plus les autrices et les auteurs vers l'écriture autobiographique. Le héros des Demi-civilisés, Max Hubert, se veut l'alter ego de 1'auteur. Dans sa présentation de l'œuvre, Guildo Rousseau souligne d'ailleurs cet aspect : 
DAVID DÉCARIE et MARIE-FRÉDÉRIQUE DESBIENS, « Le “roman-essai” (19341947). Un précurseur de l'essai littéraire au Québec?»

«Le roman vécu est chez lui le point de départ du roman fictif : la confession, l'égotisme et le libre arbitre sont en honneur dans son roman, comme le sont d'ailleurs le parti pris, la peinture de mœurs et le spiritualisme humain. » (Harvey, 1988 [1934], p. 7) La nature autobiographique du roman écrit au «je » participe à sa modernité et rapproche celui-ci de l'essai dit littéraire qui émerge, on y reviendra en conclusion, dans notre corpus.

Pour sa part, Bugnet revendique la subjectivité dès l'incipit de Siraf:

Comme à Sir Conan Doyle et à nombre d'autres hommes aujourd'hui célèbres, il m'est arrivé fort souvent de communiquer avec un esprit. Mais, à la différence du commun des spirites, je n'ai jamais eu besoin de préparer scientifiquement les séances, nul besoin non plus d'être assisté d'aucun « médium ». Je me rends bien compte que ceci est extraordinaire. Pour autant, je ne veux même pas tenter d'en offrir la moindre explication. J'estime que la meilleure manière de convaincre le lecteur consiste à lui présenter simplement les faits. Chacun peut ainsi, sans prévention, les interpréter. (1934, p. 7)

La dimension subjective y est revendiquée, notamment par l'utilisation du « je ». Le narrateur prétend que l'histoire est racontée à partir d'une expérience vécue par l'individu, sans médiation (par la science ou par une tierce personne). Il y a donc une sorte de volonté de témoignage, un désir de se présenter en tant que sujet du récit rapporté. De surcroît, cette subjectivité est accentuée par le regard du lecteur, qui va pouvoir se faire sa propre interprétation.

Le roman comporte de plus un caractère autobiographique, puisque chaque chapitre débute par une mise en scène de l'auteur travaillant ou lisant avant d'être visité par les esprits. Le troisième chapitre débute même par un extrait du journal de l'auteur daté du 15 avril 1920. (Bugnet, 1934, p. 17) 
www.revue-analyses.org, vol. 15, nº1, printemps-été 2020, p. 26-53.

Quant à elle, Lavallée se réclame du « fait vécu » dans son avertissement au lecteur : « En publiant cet ouvrage, je délivre mon âme d'un secret qui me pèse » (1936, p. 9). L'autrice veut accorder une valeur d'authenticité au récit et termine son premier chapitre sur ces mots : « Je les ai recueillies [les anecdotes] de la bouche de mon père. Le récit est fidèle ». (1936, p. 22) Et, tout au long du récit, c'est toute la relation père-fille qui se raconte, jusqu'à la mort de ce dernier. Cette dimension subjective du « fait vécu », cette prémisse de l'intime, liée à une zone autobiographique déjà présente chez Harvey et Bugnet, ne va aller qu'en s'affirmant dans les romans-essais ultérieurs.

Par bien des aspects, Désespoir de vieille fille est encore plus près du journal que de l'essai. La subjectivité est au cœur de l'œuvre : « Je m'écoute, je me parle pour m'entendre, je ne me rassasie pas de mes propres discours; je me parle de la vie. » (Tardif, 1943, p. 9) Paul Lallier est ainsi reconnaissant à l'autrice de n'avoir pas écrit un roman : «On s'attendrait à un roman ou à d'humides élégies. Il n'en est rien. Il s'agit de vie. Une âme de femme, douée d'élan de tendresse, de passion même, vieillit désespérément seule, sans avoir aimé, ni avoir été aimée selon son attente, et transcrit ses dialogues avec son cœur et sa solitude. » (1943, p. 249) La critique se montre d'autant plus sensible à cet aspect qu'il contrevient à la pudeur imposée aux femmes.

Sous le masque de leurs doubles, Laplante, Perrin et Félix, Baillargeon, Hertel et Simard se livrent avec une grande honnêteté et n'hésitent jamais à se moquer d'eux-mêmes. Baillargeon intitule un chapitre: "Pourquoi je ne fus pas lu» et Simard décoche de nombreuses flèches à son anti-héros. La subjectivité réside dans la très grande liberté que s'accordent les auteurs qui devisent à bâton rompu de tout et de rien mais également dans la primauté accordée au style, vu comme l'expression suprême de l'individualité. Ces 
DAVID DÉCARIE et MARIE-FRÉDÉRIQUE DESBIENS, « Le “roman-essai” (19341947). Un précurseur de l'essai littéraire au Québec?»

romanciers-essayistes qui citent Valéry et Proust n'ont toutefois rien de pompeux et n'hésitent pas à rapprocher leur écriture de l'oral, comme en témoigne le titre des « médisances ». Hertel et Baillargeon accordent d'ailleurs une grande place à la conversation dans leurs écrits, qui se donnent pour improvisés.

La remise en question du modèle traditionnel du roman dans les œuvres de notre corpus passe enfin par la dissolution de l'intrigue qui se décline de plusieurs façons. Des intrigues importantes apparaissent dans Les demi-civilisés, mais le roman, pour l'essentiel, est centré sur ce que les nouveaux-romanciers appelleront plus tard «l'aventure de l'écriture ». Dans le cas de Koshawika, l'intrigue du roman est transformée par l'essai en une vaste allégorie. La dissolution de l'intrigue est partielle chez Baillargeon, Hertel et Simard qui mettent en place des lambeaux de récits. Baillargeon, par la voix de son narrateur, avoue son dégoût des récits : «Je répugne à raconter des histoires. Elles sont toutes navrantes comme la vie. Elles sont toutes trop longues comme la vie. » (1945, p. 182) Le héros d'Hertel, Anatole Laplante, après avoir décrit une de ses ternes journée d'écrivain, conclut ironiquement : «Voilà ma vie. C’est une vie simple. Je viens d'écrire ceci pour éviter aux biographes un travail de recherche où ils ne trouveraient rien. » (1944, p. 30) L'intrigue disparaît complètement chez Bugnet et Tardif. Cette dissolution est programmée par l'importance que prend le versant essayistique. 
Www.revue-analyses.org, vol. 15, nº1, printemps-été 2020, p. 26-53.

\section{L'essai à l'épreuve du roman : fragmentation (formelle et thématique); caractère négatif; mise à distance des idées}

Les versants essayistiques, proprement réflexifs, des romans-essais des années 1930 et 1940 possèdent de remarquables similarités, non dans les idées elles-mêmes, mais dans les procédés utilisés pour les transmettre ${ }^{10}$.

Premièrement, à la différence des romans idéologiques d'un Groulx ou d'un Potvin, les romans ne défendent pas une thèse centrale à laquelle tout est subordonné; ils abordent des sujets multiples. Cet éclectisme s'accompagne d'une fragmentation, d'un morcèlement de la forme romanesque ${ }^{11}$. Pour Berthelot Brunet, on 1'a vu, Les demi-civilisés se présente ainsi comme un « recueil de thèses, de conversations et de rêves. » (1934, p. 4) Le roman Siraf est également dépourvu d'une thèse centrale :

Siraf est un livre curieux, où l'on discute de toutes sortes de choses, des sujets aussi disparates que le concept d'univers du point de vue des esprits, du point de vue de l'homme et du point de vue des molécules vivantes qui s'agitent dans le corps d'un poisson, et l'idée qu'une famille chinoise peut se faire de la barbarie de la civilisation occidentale en lisant les grands journaux de New-York, Chicago et Montréal. » (P.S., 1934, p. 3)

Le versant essayistique de Koshawika, de même, livre des idées en vrac : «Rien de lié dans cet ouvrage, rien de fondu. Ce sont des pensées très personnelles, jetées, ici et là, au hasard et au caprice d'une imagination débordante qui enchantera les plus difficiles » ([Anonyme], 1937, p. 2). Le morcellement des idées est encore plus important dans Désespoir de vieille fille:

\footnotetext{
${ }^{10}$ Insistons sur le caractère relatif de la division des caractéristiques communes de œuvres de notre corpus entre le roman et l'essai. L'hybridité, l'autoréflexivité et le caractère autobiographique influent ainsi sur les idées de ces œuvres.

${ }^{11}$ On rejoint, ici comme en bien des endroits, le texte de Michel Lacroix qui suit le nôtre dans ce numéro de revue.
} 
DAVID DÉCARIE et MARIE-FRÉDÉRIQUE DESBIENS, « Le “roman-essai” (19341947). Un précurseur de l'essai littéraire au Québec?»

On remarquera la composition particulière de chaque section. L'auteur commence par une exposition narrée, sorte de thème général. Puis, à sa suite, s'amorce le développement, chapelet de pensées et de sentiments détachés, certains banals, la plupart bien sentis, unifiés et harmonisés par le thème lyrique initial. (Lallier, 1943, p. 249)

L'emploi d'aphorismes rapproche de plus Tardif de Hertel, Baillargeon et Simard. Félix comporte une quarantaine de chapitres, mais ceux-ci font en moyenne deux pages. Les deuxième et troisième parties de Commerce sont constituées de courtes maximes. Cette fragmentation passe également par la division des œuvres. Dans leurs romans, les trois auteurs pratiquent une écriture qui rappelle les Essais de Montaigne (abondamment cités). Leurs romans procèdent en effet thématiquement plutôt que chronologiquement. Liées par l'époque de la Grande Noirceur, ces thématiques se recoupent souvent : on retrouve ainsi dans les Médisances un chapitre consacré aux professeurs intitulé « Mes maitres » et dans Félix un chapitre similaire intitulé « Les maîtres »; Baillargeon et Simard racontent tous deux les « soupçons odieux» que les adultes portent sur leur sexualité. Dans sa conclusion d'Anatole Laplante, curieux homme, le narrateur, conscient du caractère « en apparence épars » de son œuvre, sent l'obligation de « rassembler et lier ensemble, d'une manière certes artificielle (si nous voulons enfin qu'on tienne compte de nous dans l'histoire du roman) » (Hertel, 1944, p. 156). Baillargeon loue du reste la maxime (en désavouant le roman!) :

La vie du roman, sa principale qualité, c'est la tienne que tu perds à le lire. La maxime seule est morale. Elle seule a pour excuse sa brièveté. Elle seule enfin veut être le dernier mot. Sa tournure constitue la meilleure des preuves possibles; il n'en est pas besoin d'autres; tout ce qui allongerait la maxime l'affaiblirait. Resserrée extrêmement dans une phrase, elle n'en est que plus énergique. » (1945, p. 181-182)

Les autrices et les auteurs des romans-essais ont également en commun une vision de la société canadienne-française extrêmement négative. De façon générale, leurs romans-essais 
Www.revue-analyses.org, vol. 15, nº1, printemps-été 2020, p. 26-53.

attaquent des thèses plus qu'ils n'en défendent. Les demi-civilisés constitue dans cette optique un roman de l'anti-thèse. Bugnet résumait quant à lui sa méthode dans la note accompagnant la publication des premier et sixième chapitres de Siraf dans Le Canada Français :

Je me suis revêtu de leurs oripeaux scientifiques et, dans l'esprit de «castigare ridendo mores », je me suis diverti moi-même à montrer quelle figure prendrait leur idole (l'humanité moderne) devant des « esprits » peu célestes sans doute, dénués de mansuétude qui m'ont fait entendre quelques propos, assez inhumains, mais dont je n'affirmerai pas qu'ils soient toujours faux, comme non plus qu'ils soient toujours justes. Tout lecteur intelligent et de bonne foi pourra, je crois, par sa propre réflexion, extraire de ces propos quelques salutaires idées. (1931-1932, p. 18)

Cette méthode déstabilise visiblement la critique : «C'est une série de discussions, où l'on trouve des objections sans solutions satisfaisantes, des sophismes sans réfutation adéquate. » (P.S., 1934, p. 3)

Lavallée livre, elle-aussi, ses idées de façon essentiellement négative :

L'auteur sait voir ce qui se passe dans notre petit monde des lettres, des arts et ailleurs, et — sa loyale franchise en est bien incapable — ne se gêne pas pour peindre tous les ridicules et les travers des gens; Mlle Lavallée a-t-elle raison de nous présenter en clair-obscur aussi prononcé certains aspects de notre caractère, de notre individualité, je n'oserais pas dire de notre humanisme défaillant? D'autres avant elle l'ont fait avec moins de courtoisie et sûrement dans une langue moins française. (Bertrand, 1937, p. 6)

Il y a, chez Tardif, des dénonciations et des revendications, comme chez Lavallée, mais les idées laissent davantage de place aux mots et aux images, aux formules lapidaires et grandiloquentes. En fin de parcours, la romancière renoue de plus avec une spiritualité trempée de littérature, « avec la religion qui nous a donné le Christ de Rimbaud mourant, le Christ fort de Claudel, le Christ des fuites de Mauriac, le Christ baigné de blasphèmes par Renan, le Christ adoré par son petit-fils Ernest Psichari. » (Tardif, 1943, p. 121)

Hertel, Baillargeon et Simard poursuivent, dans une esthétique différente, le procès de la société canadienne française. La posture attribuée par un critique de l'époque au 
DAVID DÉCARIE et MARIE-FRÉDÉRIQUE DESBIENS, « Le “roman-essai” (19341947). Un précurseur de l'essai littéraire au Québec?»

personnage médisant de Baillargeon sied également à Anatole Laplante et Félix : « Claude Perrin pense; ne pense pas comme tout le monde; et même, il parait mettre du système à penser différemment, contrairement aux personnes de son milieu. C'est un homme de réaction. » (Sainte-Croix, 1945, p. 5)

Hertel, le plus savant, le plus littéraire, le plus éthéré, ne produit guère d'étincelles. Berthelot Brunet, dans son Histoire de la littérature canadienne-française, dit de celui-ci : « Révolutionnaire, François Hertel voudrait bien l'être, mais il n'y parvient pas toujours. Au fond, François Hertel est surtout un chanoine Groulx badin [...].» (Brunet, 1946, p. 106) Baillargeon, beaucoup moins prudent, décrit la férocité, la bêtise et l'ennui dans l'éducation de l'époque. Les médisances de Perrin préparent Les insolences du frère Untel. Disciple de Voltaire, Simard est tout aussi abrasif. Les deux romans mettant en scène Félix se méritent d'ailleurs la côte «Mauvais » dans la revue Lectures qui, de 1946 à 1965, identifie les lectures dangereuses. Les trois auteurs n'attaquent toutefois jamais de front, comme l'avait fait Harvey ou comme le feront les signataires du Refus global en 1948, mais pratiquent plutôt une guerre de harcèlement et d'embuscades.

Les réflexions qui sont développées sont enfin, dans la plupart des romans-essais, mises à distance. Dans Siraf, cette mise à distance des idées résulte de l'utilisation d'un appareil énonciatif complexe et d'une multiplication des points de vue, procédé qui rend le message moins univoque, comme le remarque Hervé Griffon : «La morale n’est pas prêcheuse, ne suinte ni la jaunisse, ni le larmoiement particulier aux bonzes du patriotisme, de la politique et de la littérature. Elle est toute en suggestions amusantes; ou bien elle fait voir l'absurde sans insister lourdement sur les correctifs. » (1935, p. 307) Pour certains 
Www.revue-analyses.org, vol. 15, nº1, printemps-été 2020, p. 26-53.

critiques, toutefois, le procédé menace le sens même de l'œuvre : « Sans doute ces entretiens avec les esprits donnent une certaine latitude, c'est un genre qui permet beaucoup de fantaisie. Mais enfin M. Bugnet a dû vouloir dire quelque chose. » (P.S., 1934, p. 3) Dans Koshawika, cette mise en perspective des idées par le roman est à prendre au sens propre puisque le lecteur est invité à faire des liens entre l'essai et le roman. La mise à distance des idées, dans Désespoir de vieille fille, est totale puisque le roman-essai tend vers l'abstraction. Quant à eux, Hertel, Baillargeon et Simard forgent, dans leurs cuvres, des positions énonciatives excentrées pour mieux se moquer de la bourgeoisie, de l'éducation, de la religion. Laplante et Perrin sont des écrivains obscurs et désargentés. Lepic est un étranger (Juif, Irlandais et Néo-Zélandais!) qui se fera passer pour fou afin de vivre l'expérience de l'asile. Félix est un enfant sans cesse mis à l'écart. Ajoutons que, chez ces trois écrivains, l'ironie est reine et n'épargne ni les auteurs ni les personnages ni leurs réflexions. La critique adressée par Laurent Mailhot au Félix de Jean Simard peut s'appliquer à ces trois romanciers : «Ces révoltés sans révolution, sans idéologie, sans parti, rafraîchissaient l'atmosphère duplessiste sans en troubler les marais profonds. » (1982, p. 370)

\section{Du roman-essai... à l'essai littéraire?}

Malgré leur diversité et leur originalité, les romans-essais des années 1930 et 1940 gagnent à être regroupés en vertu des nombreuses caractéristiques qu'ils partagent, tant dans leurs versants romanesques (hybridité, autoréflexivité, caractère autobiographique et dissolution de l'intrigue) que dans leurs versants essayistiques (fragmentation; caractère négatif; mise à distance des idées). Le roman-essai, à première vue, ne semble guère avoir eu de postérité. Bugnet et Lavallée cessent d'écrire; Tardif n'écrit qu'une autre œuvre (le roman La Vie quotidienne publié en 1951 à compte d'autrice). Hertel, Baillargeon et Simard chercheront 
DAVID DÉCARIE et MARIE-FRÉDÉRIQUE DESBIENS, « Le “roman-essai” (19341947). Un précurseur de l'essai littéraire au Québec?»

tous à produire par la suite de « vrais romans », ce qu'ils feront avec un certain succès mais sans véritablement trouver leur public. Comme beaucoup d'écrivains des années 1930 et 1940, ceux-ci se retrouvent en décalage dans le monde de l'après-guerre. André Gaulin rapproche, de façon convaincante, les œuvres de Baillargeon des Insolences du frère Untel de Jean-Paul Desbiens (2009, p. 42-44) ${ }^{12}$. Son article, très élogieux pour Baillargeon, montre les difficultés de l'auteur, mal-aimé de la critique et promis à l'oubli. Ces difficultés ne sontelles pas également liées au genre qu'il a pratiqué ? Une anecdote de Gaulin permet de le croire : « [S]on roman inédit Autour d'un gros bonhomme [...] aurait mérité le premier prix du Cercle du livre de France en 1949, n'eût été que les jurés... craignaient la réflexion des Français sur le fait que l'œuvre tenait à la fois du roman et de l'essai! » (2009, p. 42).

Certes, la forme du roman-essai, au sens strict, ne disparait pas tout à fait et telle ${ }^{13}$ ou telle œuvre ${ }^{14}$ peut s'y rattacher. Mais comment expliquer l'apparition dans un corpus non négligeable (soit $8 \%$ de la production romanesque de l'époque) de cette forme dans les années 1930 et 1940? Michel Lacroix, dans le texte qui suit le nôtre, émet d'intéressantes hypothèse auxquelles nous souscrivons. Nous proposons une autre piste de réflexion. Et si la forme qui cherchait à naître dans ces romans-essais n'était autre que celle de l'essai dit littéraire ${ }^{15} ?$

André Belleau, dans «Approches et situation de l'essai québécois », remarque les racines romanesques de l'essai littéraire au Québec :

\footnotetext{
${ }^{12}$ L'expression « roman-essai » apparaît dans l'un des sous-titres de son article.

${ }^{13}$ Monsieur Melville de Victor Lévy-Beaulieu par exemple.

${ }^{14}$ Le siècle de Jeanne d'Yvon Rivard (Brodeur, 2016).

${ }^{15}$ Ce qui n'exclut pas l'existence de « précurseurs » plus anciens, notamment au XIX ${ }^{\mathrm{e}}$ siècle (Simard, 1981; Lamonde, 2005).
} 
Or j'estime qu'au Québec, depuis au moins une quarantaine d'années, un bon nombre d'essais ont été produits et publiés sous le couvert du discours romanesque. Qu'on ne se méprenne pas: il ne s'agit pas des remarques, commentaires adjuvants qui constituent, dans tous les romans, le discours habituel du narrateur. Parlons plutôt de longs développements, ou de chapitres entiers, ou de fragments nombreux et disjoints, ou même d'une organisation narrative exclusivement centrée sur ce que Jean Marcel a appelé un «corpus culturel ». Encore une fois, non pas de simples «plages» idéologiques mais de considérables expansions discursives. Les Demi-civilisés de Jean-Charles Harvey offrent dès 1934 un bon exemple. (Belleau, 1980, p. 541-542)

De nombreuse caractéristiques des romans-essais que nous avons repérés sont également celles de l'essai littéraire. Étudiant la conception polyphonique de l'essai chez Belleau, Dumont montre son caractère hybride ${ }^{16}$ :

le genre de l'essai, loin de s'autonomiser, peut s'intégrer aux autres genres seconds qui ne sont pas a priori littéraires, de même qu'à un genre second littéraire comme le roman. [...] Il y aurait donc non seulement passage d'un type de genre à l'autre (du genre du discours scientifique au genre littéraire de l'essai, par exemple), mais encore une sorte de contamination dynamique entre les genres littéraires eux-mêmes (par exemple le roman et l'essai). (1997, p. 763)

Pierre Glaudes et Jean-François Louette notent son caractère autoréflexif : « l'essai suppose une écriture qui se commente, se juge sans cesse, se nuance, se critique, se contredit même. En reprenant un mot d'un commentateur de Montaigne (André Tournon), on dira qu'il procède volontiers par auto-exégèse » (2011, p. 315). Pascal Riendeau repère, au-delà de la subjectivité et de l'écriture au «je», sa «dimension autobiographique» (2005, p. 95) L'écriture de l'essai, selon Glaudes, «se refuse à la linéarité des discours persuasifs canoniques et aux structures dialectiques fermées, pour privilégier une esthétique de la fragmentation, des disparates et de la rupture, dans laquelle la déconstruction des arguments l'emporte sur l'affirmation d'une doctrine » (2002, xviii; cité dans Riendeau). Christian

\footnotetext{
${ }^{16}$ Sur les rapports de la fiction et de l'essai, voir Audet, 1999-2000; sur ceux du roman et de l'essai, voir Philippe, 2000.
} 
DAVID DÉCARIE et MARIE-FRÉDÉRIQUE DESBIENS, « Le “roman-essai” (19341947). Un précurseur de l'essai littéraire au Québec?»

Tremblay émet quant à lui l'hypothèse qu'il « incarne le discours du non-savoir [et qu']il met en valeur l'incertitude de toute connaissance » (1992, p. 11; cité dans Riendeau) André Belleau note enfin l'importance du « distancement » dans l'essai :

Pendant longtemps, notre roman nous a été donné comme se racontant tout seul, sans retour réflexif de l'énonciateur sur son énoncé au sujet des codes littéraires, de la langue, etc. L'essai au contraire, implique ce distancement car il nous propose précisément du langage sur du langage, de la culture sur de la culture, des signes sur des signes. (1980, p. 541)

Robert Vigneault, en esquissant à grands traits l'histoire de l'essai au Québec (1972), situe ses origines dans les années 1940 et voit en Baillargeon, Hertel et Simard les premiers auteurs du genre au Québec (il évacue du reste la dimension romanesque des Médisances de Claude Perrin). Dans «Petite essayistique », un article devenu canonique, André Belleau montre que cette apparition peut être envisagée comme un indice de la maturité de la littérature québécoise :

Mais l'apparition d'essayistes dans la littérature suppose une condition supplémentaire: que la teneur en culture du discours social ne se situe pas au-dessous d'un certain seuil. Car l'essayiste, lui, travaille plus spécifiquement avec le langage de la culture. Et il m'apparaît évident qu'une société où les signes de la culture sont raréfiés produira peu d'essayistes. (1983, p. 9-10)

Il réfléchit également aux rapports de l'essai et du roman et élabore sa célèbre formule : « [1]e roman est mangé par l'essai » (1983, p. 8). Le corpus ici étudié permet de donner un sens nouveau à cette manducation qu'il faudrait prendre à la lettre. Les romans-essais montrent en effet la première phase de ce processus de digestion. 


\section{Bibliographie}

\section{Romans-essais (par ordre chronologique)}

HARVEY, Jean-Charles (1988 [1934]), Les Demi-civilisés, édition critique de Guildo Rousseau, Montréal, Les Presses de l'Université de Montréal.

Bugnet, Georges (1934), Siraf, Montréal, Éditions du Totem.

LAVAlLÉE, Jeannine (1936), Koshawika, Montréal, Éditions Rénovation.

TARDIF, Thérèse (1943), Désespoir de vieille fille, Montréal, Éditions de l'Arbre.

Hertel, François (1944), Anatole Laplante, curieux homme, Montréal, Éditions de l'Arbre.

Baillargeon, Pierre (1945), Les médisances de Claude Perrin, Montréal, Parizeau.

Baillargeon, Pierre (1947) Commerce, Montréal, Éditions Variétés, Dussault et Péladeau.

HERTEL, François (1947) Journal d'Anatole Laplante, Montréal, Éditions Serge Brousseau.

SiMARD, Jean (1947), Félix, livre d'enfant pour adultes, Montréal, Éditions Variétés, Dussault et Péladeau.

\section{Autres œuvres citées (par ordre alphabétique)}

[Anonyme] (1934), « Désespoir de vieille fille », Le Jour, vol. 6, nº 36, p. 3.

[Anonyme] (1937), « Koshawika », En avant!, vol 1, nº 1, p. 2.

AUDET, René (décembre 1999-février 2000), « La fiction à l'essai », colloque en ligne Fabula « Frontières de la fiction », http://www.fabula.org.

Belleau, André (1980), « Approches et situation de l'essai québécois », Voix et images, vol. $5, \mathrm{n}^{\mathrm{o}} 3$, p. 537-543.

Belleau, André (1983), « Petite essayistique », Liberté, vol. 25, nº 6, p. 7-10.

Bertrand, Camille (23 janvier 1937), « Koshawika », Le Devoir, vol. 28, nº 17, p. 6.

BIRON, Michel, François Dumont et Élisabeth Nardout-Lafarge (2007), Histoire de la littérature québécoise, Montréal, Boréal. 
DAVID DÉCARIE et MARIE-FRÉDÉRIQUE DESBIENS, « Le “roman-essai” (19341947). Un précurseur de l'essai littéraire au Québec?»

BRODEUR, Andréanne (2016), « Le dialogue entre l'essai et le roman dans Le Siècle de Jeanne », mémoire de maîtrise, Université de Montréal.

BRUNET, Berthelot (1934a), « Quand le Québec se dessale », L’Ordre, vol. 1, n 39, p. 4.

BRUNET, Berhelot (1934b), « Le philosophe d'Alberta », L'Ordre, vol. 1, n² 222, p. 4.

BRUNET, Berhelot (1946), Histoire de la littérature canadienne-française, Montréal, Éditions de l'Arbre.

Bugnet, Georges (1931-1932), « Siraf », Le Canada Français, vol. 19, p. 18.

ChARTIER, Daniel (2000), L'émergence des classiques. La réception de la littérature québécoise des années 1930, Montréal, Fides, coll. « Nouvelles études québécoises ».

DANTIN, Louis, (8 avril 1965), « Exclusif au Devoir. Vingt lettres inédites de Louis Dantin à Louvigny de Montigny », Le Devoir, vol. 56, no 82, p. 23-24.

DÉCARIE, David (2016), « L'évolution du roman urbain (1934-1945) : du roman bourgeois au roman du peuple », Voix et Images, vol. 41, n 2 , p. 21-33.

DumONT, François (1997), « Le fonds des formes : la dynamique des genres chez André Belleau », Revue belge de philologie et d'histoire, vol. 75, n 3, p. 761-769.

GAulin, André (2009), « Essais pré-politiques : des Médisances aux Insolences », Québec français, $\mathrm{n}^{\mathrm{o}} 153$, p. 42-44.

GiguÈRE, Andrée-Anne (2016), « Les écrivains de La Relève et la pensée romanesque : critique et pratique du roman chez Robert Charbonneau, Robert Élie, Jean Le Moyne et Hector de Saint-Denys Garneau », thèse de doctorat, Québec, Université Laval, 2016.

GRIFFON, Hervé (mai 1935), « Siraf », L'action nationale, vol. V, nº 5, p. 307.

GUAY, Élyse et Michel Lacroix (2016), « Saillies et paradoxes : Amérique française et 1'ethos du moraliste bouffon ». Voix et Images, vol 41, n 2, p. 67-81.

GLAudes, Pierre et Jean-François louette (2011), L'essai, Paris, Armand Colin.

GLAUDES, Pierre, «Introduction », dans : Pierre glaudes (dir.), L'essai : métamorphoses d'un genre, Toulouse, Presses universitaires du Mirail, 2002, p. i-xxvi.

LALLIER, Paul (1943), « Désespoir de vieille fille », Relations, nº 33, p. 249. 
LAMONDE, Yvan (2005), « L'essai littéraire au Québec au XIXe siècle : le problème de sa définition et du statut de la prose d'idées "), Les Cahiers des dix, n 59, p. 21-54.

MAiLhot, Laurent (1982), «Félix », dans Maurice Lemire (dir.), Dictionnaire des œuvres littéraires du Québec, tome III : 1940-1959, Montréal, Fides, p. 370.

PARIZEAU, Lucien (1934), « Le roman de Jean-Charles Harvey », $L^{\prime} O$ rdre, vol. 1, n 44, p. 1.

PhilipPe, Gilles, dir. (2000), Récits de la pensée. Études sur le roman et l'essai, Centre d'études du roman et du romanesque, Université de Picardie - Jules Verne, Paris, Sedes.

P. S. (19 décembre 1934), «Que veut-il dire? », Le Devoir, vol. 25, no 291, p. 3.

R. D. (1938), « Mea Culpa, Koshawika », L'action nationale, vol. 11, nº 3, p. 264.

RIENDEAU, Pascal (2005), « La rencontre du savoir et du soi dans l'essai », Études littéraires, vol. 37, no 1, p. 91-103.

ROBERT, Lucie (2011), « De La Vie littéraire à La Vie culturelle. "Vie”, avez-vous dit ?», Revue d'histoire littéraire de la France, vol. CXI, no 1, p. 89-105.

SAinte-Croix, A. (11 août 1945), « Portrait d'un original », Le Jour, vol. 8, no 48, p. 5.

SimARD, Sylvain (1981), « L'essai québécois au XIXe siècle », Voix et Images, vol. 6, n 2, p. 261-268.

TREMBlay, Christian (1992), Le processus de transformation cognitive. Le cas de la thèse de doctorat, thèse de doctorat, Québec, Université Laval, 1992.

VignEAUlT, Robert (1972), «L'Essai québécois : la naissance d'une pensée » Études littéraires, vol. 5, $\mathrm{n}^{\circ}$ 1, p. 59-73.

\section{Notices biobibliographiques}

Professeur au département d'études françaises de 1'Université de Moncton et membre de l'équipe de la série La vie littéraire au Québec, David Décarie est un spécialiste du roman français et québécois du XXe siècle. Publications récentes : Métro-tout-nerfs-railsmagiques, Les transports dans l'œuvre de Louis-Ferdinand Céline, éditions 8, 2018; avec Lori Saint-Martin : Germaine Guèvremont, Le Cycle du Survenant I, Presses de l'Université de Montréal, 2017. 
DAVID DÉCARIE et MARIE-FRÉDÉRIQUE DESBIENS, « Le "roman-essai” (19341947). Un précurseur de l'essai littéraire au Québec?»

Après avoir rédigé un mémoire sur les dernières lettres de Chevalier de Lorimier, MarieFrédérique Desbiens a collaboré à des éditions critiques sur l'histoire et les écrits des Patriotes. Elle a également prononcé plusieurs communications au Québec et en Europe sur ces questions ainsi que sur le roman historique, sujet de la recherche postdoctorale qu'elle a menée au CNRS à Paris de 2005 à 2007. En 2019, elle s'est vu décerner le prix Jean-ÉthierBlais pour son ouvrage Le premier romantisme au Canada. Entre engagement littéraire et politique, issu de sa thèse de doctorat. Cosignataire de La vie littéraire au Québec, elle a été coordonnatrice de cette équipe pendant plus de 10 ans avant de devenir responsable de programmes au Fonds de recherche du Québec - Société et culture. 